\title{
Proficiency Monitoring of Allergen-Specific IgE macELISA - 2019
}

\author{
Kevin Enck ${ }^{1}$, Kenneth Lee ${ }^{1}$, Karen Blankenship ${ }^{1}$, Brennan McKinney ${ }^{1}$, Majda Globokar ${ }^{2}$, Elizabeth Roth ${ }^{3}$, Janice Greenwood ${ }^{4}$, \\ Santiago Cerrato ${ }^{5}$, Laurent Drouet ${ }^{6}$ and Cecilia Tambone ${ }^{7}$
}

1Stallergenes Greer, 639 Nuway Circle, Lenoir, North Carolina, USA

2IDEXX GmbH, Mörikestraße 28/3, D-71636 Ludwigsburg, Germany

${ }^{3}$ IDEXX Laboratories, Inc. One IDEXX Drive, Westbrook, Maine, USA

${ }^{4}$ IDEXX Laboratories, Inc. 1345 Denison Street, Markham, Ontario L3R 5V2, Canada

${ }^{5}$ LETI s.l.u., Avda Cerdanyola, 92, 08172 Sant Cugat, Barcelona, Spain

${ }^{6}$ Ceva Biovac, 6 rue Olivier de Serres, BP 10061 Angers Technopole, 49071 Beaucouzé, France

${ }^{7}$ Agrolabo S.p.A.,Via Masero, 59, 10010 Scarmagno (TO), Italy

*Corresponding Author: Kevin Enck, Stallergenes Greer, 639 Nuway Circle, Lenoir, NC 28645, USA; Tel: +18287597345; Fax: +18287547306; E-mail: kevin.enck@ stallergenesgreer.com

Received: November 17, 2019; Accepted: December 03, 2019; Published: December 12, 2019;

\begin{abstract}
This study was designed to evaluate the reproducibility of a macELISA for the detection of allergen-specific IgE in dogs and cats. Nine different individuals across seven separate affiliated laboratories evaluated 21 predefined sera samples in a single blinded fashion. For evaluations completed by multiple operators, the average inter-operator variance was calculated to be $3.7 \%$ (range $=1.5 \%-4.7 \%$ ). The average intra-assay variance among reactive assay calibrators in all laboratories was $4.1 \%$ (range $=0.3-11.9 \%$ ). The overall inter-assay inter-laboratory variance evident with reactive calibrators was consistent among laboratories and averaged 10.4\% (range $4.4-13.0 \%$ ). All laboratories yielded similar profiles and magnitudes of responses for replicate unknown samples; dose response profiles observed in each of the laboratories were indistinguishable. Correlation of EAU observed for individual allergens between and among all laboratories was strong $(r>0.90, p<0.001)$. Collectively, the results demonstrated that the macELISA for measuring allergen-specific IgE is reproducible, and documents that consistency of results can be achieved not only in an individual laboratory, but among different operators and between laboratories using the same macELISA.
\end{abstract}

Keywords: IgE, ELISA, Proficiency, Atopy, Allergy, Immunotherapy, Cross-reactive Carbohydrate

\section{Introduction}

Stallergenes Greer maintains a proficiency monitoring program for laboratories that routinely run a monoclonal antibody cocktail based enzyme-linked immunosorbent assay (macELISA) for evaluation of allergen-specific IgE in serum samples [1-4]. The foundation for this program is based in the desire for inter-laboratory standardization and quality control measures that ensure the uniformity, consistency, and reproducibility of results among laboratories that perform the assays. This program, now in its tenth year, is designed to periodically evaluate the proficiency of laboratories and ensures that individual operators yield consistent and reproducible results. The first proficiency evaluations documented that inter-laboratory standardization and quality control measures in the veterinary arena are on the immediate forefront and that uniformity, consistency, and reproducibility of results between laboratories is achievable [2]. Similarly, reproducibility of results among ten different laboratories was documented in the proficiency evaluations subsequently completed [2-4]. The results presented herein summarize the comparative results observed in the affiliate laboratories for the most recent proficiency evaluations that were completed in 2019 .

\section{Materials and Methods}

All serum samples, buffers, coated wells, calibrator solutions, and other assay components were aliquants of the respective lots of materials manufactured at Stallergenes Greer's production facilities (located in Lenoir, NC, USA) and supplied as complete kits to the participating laboratories along with the exact instructions for completing the evaluations.

\section{Participating Laboratories}

Seven independent Veterinary Reference Laboratories (VRLs) participated in the 2019 proficiency evaluation of macELISA. Participating laboratories included three separate IDEXX laboratories located in Memphis, Tennessee, Ludwigsburg, Germany, and Markham, Ontario Canada. Other affiliated European laboratories that participated in this evaluation included Agrolabo (Scarmagno, Italy), Laboratories LETI (Barcelona, Spain), and Ceva Biovac (Beaucouzé, France). Stallergenes Greer Laboratories (Lenoir, NC) served as the prototype for evaluation of macELISA; the 2019 evaluations included results reported by three separate and independent operators. Because the performance characteristics of macELISA in Stallergenes Greer's 
VRL have been well documented for use over an extended period [1-4], all results observed in the other participating laboratories were compared directly with the results observed in Stallergenes Greer's reference laboratory.

\section{Serum Samples}

Separate pollen and mite reactive sera pools as well as nonreactive sera pools were prepared from cat and dog serum samples that previously had been evaluated using the macELISA for detection of allergen-specific IgE in dogs and cats. The allergen-specific reactivity of each sera pool ranged from nonreactive to multiple pollen or mite reactivity's. These sera pools and admixtures of the pools were used to construct a specific group of samples that exhibited varying reactivity to the allergens included in the evaluation panel. Eighteen samples were included in the blinded evaluation conducted by each laboratory. Two known pollen reactive control samples and one nonreactive control sample were also included; replicates of these identical samples were included as unknown blinded samples. Also included in the array of samples was a five tube three-fold serial dilution of a highly pollen-reactive pool, diluted into non-reactive sera, which served to document the dose response evident within the assay. All samples were stored at $-20^{\circ} \mathrm{C}$ for the interim between testing.

\section{Calibrators}

Grass pollen reactive calibrator solutions of predetermined reactivity in the macELISA were prepared as three-fold serial dilutions of a sera pool highly reactive to most pollen allergens. Replicates of each were evaluated in each assay run and served as a standard response curve for normalizing results observed with the various samples. All results were expressed as ELISA Absorbance Units (EAU) which are background-corrected observed responses expressed as milli absorbance.

\section{Buffers}

The buffers used throughout have been previously described [1-4], and included: a) well coating buffer: $0.05 \mathrm{M}$ sodium carbonate bicarbonate buffer, $\mathrm{pH}$ 9.6; b) wash buffer: phosphate buffered saline (PBS), pH 7.4, containing $0.05 \%$ Tween 20 , and $0.05 \%$ sodium azide; c) reagent diluent buffer: PBS, $\mathrm{pH} 7.4$, containing $1 \%$ fish gelatin, $0.05 \%$ Tween 20 and $0.05 \%$ sodium azide. Unique to this year's evaluation was the inclusion of a serum diluent that contains an inhibitor of antibodies that are cross reactive to carbohydrate determinants (CCD). The inhibitor for the CCD (BROM-CCD) is a preparation containing the carbohydrate components present in bromelain, which was prepared in house and remains a proprietary product of Stallergenes Greer (Lenoir, NC, USA) [5]. The serum diluent consists of the reagent diluent with BROM-CCD added at a concentration of $2.5 \mathrm{mG} / \mathrm{mL}$.

\section{Allergen Panel}

The allergen panel was a 24 allergen composite derived from the array of allergens that are included in the specific panels routinely evaluated in the various laboratories; the composite allergen panel consisted of 4 grasses, 6 weeds, 6 trees, 5 mites, and 3 fungi. The protocol for coating and storage of wells has been previously described [1-4].

\section{Sample Evaluations - macELISA}

The operational characteristics and procedures for the macELISAs have been previously described [1-4]. Following incubation of allergen coated wells with an appropriately diluted serum sample, allergen-specific IgE is detected using a secondary antibody mixture of biotinylated monoclonal anti-IgE antibodies, streptavidin alkaline phosphatase as the enzyme conjugate, and p-nitrophenylphosphate (pNPP) as substrate reagent. Specific IgE reactivity to the allergens is then estimated by determining the absorbance of each well measured at $405 \mathrm{nM}$ using an automated plate reader. All results are expressed as ELISA Absorbance Units (EAU), which are background-corrected observed responses expressed as milli absorbance [1].

\section{Statistics}

A coefficient of variation was calculated as the ratio of standard deviation and means of the responses observed for the calibrator solutions within different runs in multiple laboratories. Pearson's correlation statistic was used for inter-laboratory comparison among individual allergens.

\section{Results}

The assay variance (\% CV) observed with the calibrator solutions in the different laboratories are presented in Table 1 and are representative of the assay reproducibility in the various laboratories. The average intra-assay \% CV among positive calibrators (\#1-4) was $4.1 \%$ (range $=0.3-11.9 \%$ ); differences among laboratories or between assays and within assay runs were not detected. No substantial difference in results among various operators were revealed. The average inter-operator variance documented for Stallergenes Greer technicians was calculated to be $3.1 \%$ (range $=0.3 \%-5.0 \%$ ). While the allergens and serum are the same as the previous 2 proficiency tests, the incorporation of CCD inhibitors precludes direct comparison to prior tests. The results of the current evaluation (Table 1) show that the inter-assay variance among positive calibrators for all laboratories included in this evaluation was calculated to be $10.4 \%$ (range $=5.5-$ $13.0 \%$ ). The intra-assay variability of the negative calibrator \#5 was $3.7 \%$ (range $0.2-7.6 \%$ ), while the background ODs had the highest intra-assay variance overall (average 5.5\%; range 0.1-13.9\%). A negative response is classified as anything with an EAU below 150 . Any analysis of results below this threshold, especially when looking at \%CV and relative differences, should be done so cautiously.

To evaluate the strength of association with the magnitude of EAU results observed for each allergen among the different laboratories a Pearson's correlation coefficient was determined (Microsoft Excel 2016) for each laboratory pair. Because the macELISA is designed to yield comparable responses in dog and cat samples, comparison of results among affiliate laboratories included both cat and dog samples as a single population of sera samples. These results (Table 2) demonstrate that very high inter-laboratory correlation $(r>$ $0.90 ; \mathrm{p}<0.001)$ is evident between the results observed in Stallergenes Greer's laboratory and those observed in six affiliate laboratories for 
all mites and pollen allergens. The correlation (Pearson's) of results observed with the fungal allergens within or between any of the testing laboratories was also substantial. However, the majority of results for the fungal allergens fell within the lower range of reactivity or within the negative range of the response curve (<150 EAU). Consequently, the correlation of results among laboratories for the fungal allergens was somewhat less than the correlation evident with the mite and pollen allergens. The overall correlation of results observed in the various laboratories are summarized in Table 3; a very strong correlation was demonstrated between and among the results of the participating laboratories.

Table 1. Calculated variance of macELISA calibrator solutions observed with different laboratory runs by multiple operators during the 2019 Proficiency evaluation.

\begin{tabular}{|c|c|c|c|c|c|c|c|}
\hline \multirow[b]{2}{*}{ Variance } & \multirow[t]{2}{*}{$\mathbf{N}$} & \multicolumn{5}{|c|}{ Calibrator $\% \mathrm{CV}^{*}$} & \multirow{2}{*}{$\frac{\mathrm{BG}^{\dagger}}{\% \mathrm{CV}}$} \\
\hline & & $\# 1$ & $\# 2$ & $\# \mathbf{3}$ & $\# 4$ & $\# \mathbf{5}$ & \\
\hline Inter-Laboratory & 266 & 5.5 & 12.5 & 13.0 & 10.8 & 9.0 & 12.1 \\
\hline Inter-Assay (Stallergenes Greer) & 124 & 1.5 & 4.1 & 4.7 & 4.4 & 3.5 & 4.2 \\
\hline \multicolumn{8}{|l|}{ Intra-Assay } \\
\hline Stallergenes Greer \#1 & 28 & 1.3 & 3.8 & 4.4 & 4.2 & 3.8 & 4.2 \\
\hline Stallergenes Greer \#2 & 28 & 1.5 & 4.1 & 5.0 & 4.1 & 2.9 & 5.2 \\
\hline Stallergenes Greer \#3 & 28 & 1.3 & 3.8 & 4.4 & 4.2 & 3.8 & 4.2 \\
\hline Stallergenes Greer \#4 & 28 & 3.5 & 2.1 & 0.8 & 0.3 & 0.2 & 0.1 \\
\hline IDEXX Memphis & 28 & 1.3 & 3.8 & 4.4 & 4.2 & 3.8 & 4.2 \\
\hline IDEXX Canada & 28 & 1.3 & 1.8 & 2.1 & 2.5 & 2.8 & 13.9 \\
\hline IDEXX Germany & 28 & 1.3 & 3.8 & 4.4 & 4.2 & 3.8 & 4.2 \\
\hline Agrolabo & 28 & 3.8 & 11.8 & 11.9 & 10.5 & 7.6 & 5.9 \\
\hline Biovac & 28 & 6.3 & 4.7 & 7.4 & 7.1 & 4.8 & 5.1 \\
\hline LETI & 28 & 2.0 & 5.5 & 4.5 & 5.5 & 3.4 & 7.8 \\
\hline
\end{tabular}

* Calibrator \#1 is prepared as a dilution of a sera pool which is highly reactive to grass pollen allergens; calibrator \#5 is a dilution of a negative sera pool. Calibrators \#2 - \#4 are prepared as a serial 3-fold dilution of calibrator \#1.

$\dagger$ Background responses observed with diluent in place of serum sample.

There is no compelling evidence that the level of allergen-specific IgE correlates with severity of clinical disease [6-9]. However, an evaluation that purports to measure allergen-specific IgE should have a reduction in signal that is directly proportional to the dilution factor of the test ligand [10]. For an evaluation of the dose response in this ELISA, a five tube three-fold serial dilution of a highly pollen-reactive dog sera pool was included as unknown independent samples. To be expected, the magnitude of responses observed in each laboratory was reduced in direct proportion to dilution (data not shown). Results from the final tube in the dilution scheme yielded results that were indistinguishable from negative responses, indicating a dilution extinction of detectable response.

\section{Discussion}

The results of the present study demonstrate that the variability between and among the affiliate laboratories and technicians are indistinguishable from the results evident within and between runs completed in the laboratory of Stallergenes Greer. The intra-assay variance observed with the positive calibrators evident among the various runs within each of the laboratories remains relatively low and indistinguishable among the various laboratories. Likewise, the interassay variance within each laboratory remained relatively constant and the results from all laboratories were demonstrably similar and the $\mathrm{CV}$ of the positive responses was relatively constant across the entire range of reactivity tested. Thus, we conclude that any and all laboratories and technicians are equally proficient in providing consistent results for all allergens tested and the results are well within the acceptable variance limits ( $\pm 20 \%)$ established for this assay [1].

Over the past ten years we have documented the reproducibility and robust character of the macELISA. In our most recent report [4], we document that comparable reproducibility of results can be achieved for a panel of identical sera samples when evaluated across multiple years. For the present study we document that inclusion of BROM-CCD inhibitor in our serum diluent [5] does not affect the intra-assay or inter-assay variance of the test. Incorporation of a CCD 
Kevin Enck (2019) Proficiency Monitoring of Allergen-Specific IgE macELISA - 2019

Table 2. Inter-laboratory correlation of macELISA results observed with individual allergens in Stallergenes Greer Laboratory and the results observed in the individual affiliate laboratories.

\begin{tabular}{|c|c|c|c|c|c|c|}
\hline \multirow{4}{*}{ Allergens } & \multicolumn{6}{|c|}{ Inter-Laboratory Coefficient of Correlation } \\
\hline & \multicolumn{6}{|c|}{ Stallergenes Greer vs } \\
\hline & IDEXX & IDEXX & IDEXX & & & \\
\hline & Memphis & Ludwiasburg & Markham & Biovac & Agrolabo & LETI \\
\hline \multicolumn{7}{|l|}{ Mites } \\
\hline Acaris siro & 1.000 & 1.000 & 0.999 & 0.973 & 0.983 & 0.981 \\
\hline Dermatophagoides farinae & 1.000 & 0.995 & 1.000 & 1.000 & 1.000 & 1.000 \\
\hline Dermatophagoides pteronyssinus & 0.999 & 0.999 & 0.994 & 0.971 & 0.914 & 0.989 \\
\hline Lepidoglyphus destructor & 1.000 & 1.000 & 0.993 & 0.986 & 0.933 & 0.993 \\
\hline Tyrophagus putrescentiae & 1.000 & 1.000 & 0.998 & 0.981 & 0.991 & 0.982 \\
\hline \multicolumn{7}{|l|}{ Grasses } \\
\hline June Grass (Poa pratensis) & 1.000 & 1.000 & 0.996 & 0.981 & 0.981 & 0.992 \\
\hline Meadow fescue (Festuca pratensis) & 0.998 & 0.983 & 0.999 & 0.999 & 0.999 & 0.999 \\
\hline Orchard Grass (Dactylis glomerata) & 1.000 & 1.000 & 0.995 & 0.994 & 0.967 & 0.990 \\
\hline Perennial Rye (Lolium perenne) & 1.000 & 1.000 & 0.997 & 0.978 & 0.970 & 0.990 \\
\hline \multicolumn{7}{|l|}{ Trees } \\
\hline Birch (Betula pendula) & 1.000 & 1.000 & 0.989 & 0.986 & 0.951 & 0.965 \\
\hline Cypress (Cupressus sempervirens) & 0.999 & 0.999 & 0.976 & 0.973 & 0.983 & 0.969 \\
\hline Hazelnut (Corylus avellana) & 1.000 & 1.000 & 0.997 & 0.960 & 0.896 & 0.949 \\
\hline Olive (Olea europaea) & 0.999 & 0.995 & 0.998 & 0.999 & 0.999 & 0.999 \\
\hline Populus mix (P. nigra, P. tremula, P. alba) & 1.000 & 1.000 & 0.998 & 0.975 & 0.932 & 0.981 \\
\hline Willow Black (Salix discolor) & 1.000 & 1.000 & 0.999 & 0.977 & 0.921 & 0.978 \\
\hline \multicolumn{7}{|l|}{ Weeds } \\
\hline English Plantain (Plantago lanceolata) & 0.999 & 0.999 & 0.998 & 0.980 & 0.935 & 0.977 \\
\hline Lambs Quarter (Chenopodium album) & 0.989 & 0.985 & 0.998 & 0.999 & 0.999 & 0.999 \\
\hline Mugwort (Artemisia vulgaris) & 1.000 & 1.000 & 0.996 & 0.990 & 0.940 & 0.989 \\
\hline Pellitory (Parietaria officinalis) & 1.000 & 1.000 & 0.997 & 0.983 & 0.944 & 0.958 \\
\hline Ragweed (Ambrosia trifida, A. artemisiifolia) & 0.999 & 0.999 & 0.994 & 0.998 & 0.988 & 0.993 \\
\hline Sheep Sorrel (Rumex acetosella) & 1.000 & 1.000 & 0.994 & 0.986 & 0.964 & 0.989 \\
\hline \multicolumn{7}{|l|}{ Fungi } \\
\hline Alternaria alternata & 0.995 & 0.995 & 0.987 & 0.977 & 0.971 & 0.942 \\
\hline Aspergillus fumigatis & 0.999 & 0.997 & 0.999 & 0.998 & 0.998 & 0.998 \\
\hline Cladosporium herbarum & 0.997 & 0.997 & 0.969 & 0.934 & 0.952 & 0.977 \\
\hline Overall & 1.000 & 0.998 & 0.997 & 0.972 & 0.981 & 0.987 \\
\hline
\end{tabular}

*Pearson Correlation Coefficient (r); Good Correlation $(r>0.8, \mathrm{p}<0.001)$ 
Table 3. Inter-laboratory correlation of macELISA results observed among individual affiliate laboratories.

\begin{tabular}{|c|c|c|c|c|c|c|c|}
\hline \multirow[b]{2}{*}{ Laboratory } & \multicolumn{7}{|c|}{ Inter-laboratory Coefficient of Correlation* } \\
\hline & $\begin{array}{c}\text { Stallergenes } \\
\text { Greer }\end{array}$ & $\begin{array}{c}\text { IDEXX } \\
\text { Memphis }\end{array}$ & $\begin{array}{l}\text { IDEXX } \\
\text { Germany }\end{array}$ & $\begin{array}{l}\text { IDEXX } \\
\text { Canada }\end{array}$ & $\begin{array}{c}\text { Ceva } \\
\text { Biovac }\end{array}$ & Agrolabo & LETI \\
\hline Stallergenes Greer & 1 & 0.992 & 0.993 & 0.997 & 0.972 & 0.981 & 0.986 \\
\hline IDEXX Memphis & 0.992 & 1 & 0.991 & 0.990 & 0.971 & 0.983 & 0.990 \\
\hline IDEXX Germany & 0.993 & 0.991 & 1 & 0.994 & 0.960 & 0.979 & 0.988 \\
\hline IDEXX Canada & 0.997 & 0.990 & 0.994 & 1 & 0.964 & 0.980 & 0.986 \\
\hline Biovac & 0.972 & 0.971 & 0.960 & 0.964 & 1 & 0.979 & 0.972 \\
\hline Agrolabo & 0.981 & 0.983 & 0.979 & 0.980 & 0.979 & 1 & 0.983 \\
\hline LETI & 0.986 & 0.990 & 0.988 & 0.986 & 0.972 & 0.983 & 1 \\
\hline
\end{tabular}

*Pearson Correlation Coefficient (r); Good Correlation $(\mathrm{r}>0.8, \mathrm{p}<0.001)$

inhibitor has been shown to be critical for reduction in false positives that occur due to the binding of certain IgE to these carbohydrate groups that are common among pollen allergens, thus leading to potential increases in signal for the assay.

The positive response threshold for this assay has repeatedly been documented to be 150 EAU [1-4]. Simply stated, samples shown to have values less than 150 EAU should be considered non-reactive to a given allergen; samples with values in the 150-4000 EAU range can be considered to exhibit specific IgE reactivity that is proportional to serum concentration. We have previously documented that a threefold increase in allergen-specific IgE content is required to affect an approximate two-fold increase in EAU. If we assume a relative concentration of 1 is required to effect an EAU signal of 150 then the relative concentration of allergen-specific IgE evident in the range of 150-300 EAU will be approximately 1-3, the relative concentration in the 301-600 EAU range will be 3-9, the 601-1200 EAU range will be 9-27, the 1200-2400 EAU range will be 27-81, while the relative concentration of IgE needed to effect a maximal signal will be greater than 150 . This being the case, it is unlikely that a highly reactive serum sample will be detected as non-reactive at a 1:5 dilution. The variance evident in the low level range of responses dictates that true borderline positive samples might be identified as false negative responses and this tendency might compound the likelihood of false negative responses. However, a serum sample at a 1:5 dilution makes detection of false positive results seem rather remote. Further, EAU values in the range of $0-150$ cannot be differentiated and comparison of the reproducibility of results within this range is moot (i.e. beyond the scope of the assay), except they are defined as negative responses. Only when EAU values are within the range of defined reactivity (150 - 4000 EAU) can the magnitude of response be used to compare the reproducibility of an assay.

We have demonstrated a continued reliability and reproducibility of our macELISA with the open publication of our proficiency testing procedures and results. We encourage other groups to determine and document similar findings; however, we emphasize the importance of identifying results below the cutoff of 150 EAU merely as non-reactive and consequently negative responses. The reproducibility of the assay for these responses need to be defined only as negative and their numerical values become meaningless; comparison of EAU values are meaningful for reactive samples only. Because the magnitude of specific responses is dependent on the concentration of allergenspecific IgE that can span a wide range, a better means of comparison of repeat results for individual samples in an assay of this sort is to evaluate the correlation (perhaps Pearson statistic) of results that might exist.

The lack of a regulatory mandated quality assurance program for serum allergen-specific IgE testing in companion animals, that independently monitors performance of all laboratories and assay formats, prompts Stallergenes Greer to accept the responsibility for continued evaluation of laboratories that routinely use the company's assays. Information presented herein documents the continued commitment of Stallergenes Greer and its affiliate laboratories to providing a stream of information relating these results to the veterinary community.

\section{Authors Contributions}

Kevin Enck and Kenneth Lee contributed to the conception and design of the study; contributed to the acquisition, analysis, and interpretation of data; and drafted the manuscript. Karen Blankenship and Brennan McKinney manufactured all components used throughout the evaluation and contributed to acquisition of the data. Gerhard Kern, Elizabeth Roth, Janice Greenwood, Santiago Cerrato, Laurent Drouet, and Cecilia Tambone contributed to acquisition of the data. All authors gave final approval and agree to be accountable for all aspects of the work in ensuring that questions relating to the accuracy or integrity of any part of the work are appropriately investigated and resolved. 


\section{Funding}

Funding for this study was provided by Stallergenes Greer.

\section{References}

1. Lee KW, Blankenship KD, McCurry ZM, Esch RE, DeBoer DJ, et al. (2009) Performance characteristics of a monoclonal antibody cocktail-based ELISA for detection of allergen-specific IgE in dogs and comparison with a high affinity $\operatorname{IgE}$ receptor-based ELISA. Vet Dermatol 20: 157-164. [crossref]

2. Lee KW, Blankenship KD, McCurry ZM (2012) Reproducibility of a Monoclonal Antibody Cocktail Based ELISA for Detection of Allergen Specific IgE in Dogs: Proficiency Monitoring of macELISA in Six US and European Laboratories. Vet Immunol Immunopathol 148: 267-275.

3. Lee KW, Blankenship K, McKinney B, Kern G, Buch J, et al. (2015) Proficiency monitoring of monoclonal antibody cocktail-based enzyme-linked immunosorbent assay for detection of allergen-specific immunoglobulin $\mathrm{E}$ in dogs. Journal of Veterinary Diagnostic Investigation 27: 461-469.

4. Lee K, Blankenship K, McKinney B, Kern G, Roth E, et al. (2018) Proficiency Monitoring of Allergen Specific IgE macELISA - 2018. Integr J Vet Biosci 2: 1-6.

5. Lee KW, Blankenship KD, McKinney BH, Morris DO (2019) Detection and Inhibition of IgE for cross-reactive carbohydrate determinants evident in an enzyme linked immunosorbent assay for detection of allergen specific $\operatorname{IgE}$ in the serum of dogs and cats. Vet Dermatol: Submitted

6. DeBoer DJ, Hillier A (2001) The ACVD task force on canine atopic dermatitis (XVI): laboratory evaluation of dogs with atopic dermatitis with serum-based "allergy" tests. Vet Immunol Immunopathol 81: 277-287. [crossref]

7. Gorman NT, Halliwell REW (1989) Atopic Diseases. In: Halliwell REW, Gorman NT (ed). Veterinary Clinical Immunology, WB Saunders, Philadelphia. Pg No: $232-252$.

8. Griffin CE, DeBoer DJ (2001) The ACVD task force on canine atopic dermatitis (XIV): clinical manifestation of canine atopic dermatitis. Vet Immunol Immunopathology 81: 255-69.

9. Griffin CE, Hillier A (2001) The ACVD task force on canine atopic dermatitis (XXIV): allergen-specific immunotherapy. Vet Immunol Immunopathol 81: 363383. [crossref]

10. Tijssen P (1993) Processing of data and reporting of results of enzyme immunoassays. In: Burdon, RH, van Knippenberg PH (eds.). Practice and Theory of Enzyme Immunoassays, Elsevier, Amsterdam. Pg No: 385-421.

\section{Citation:}

Enck K, Lee K, Blankenship K, McKinney B, Globokar M, et al. (2019) Proficiency Monitoring of Allergen-Specific IgE macELISA-2019. Integr J Vet Biosci Volume 3(3): $1-6$. 